\title{
Improvement in Hyperexpression with a Combination of Truncated Scmp Promoter and Streptomyces Lividans
}

\section{Misugi Uraji}

Graduate school of Science, Technology and Innovation

Lingli Yang

RIBS, Okayama

Tadashi Hatanaka ( $\sim$ hatanaka@bio-ribs.com)

RIBS, Okayama https://orcid.org/0000-0003-0325-3954

\section{Research Article}

Keywords: Streptomyces, secretion, protein expression, promoter, extracellular protein

Posted Date: February 3rd, 2022

DOI: https://doi.org/10.21203/rs.3.rs-1313048/v1

License: (c) (i) This work is licensed under a Creative Commons Attribution 4.0 International License.

Read Full License 


\section{Abstract}

We have previously reported a powerful promoter from the Streptomyces cinnamoneus TH-2 strain named 'scmp' and created an expression vector of pTONA5a based on plJ702 for expression using $S$. lividans (Hatanaka et al. (2008) Protein Expr Purif 62: 244-248). The full-length scmp promoter sequence consists of $424 \mathrm{bp}$ upstream of a metalloendoprotease gene in the $S$. cinnamoneus TH-2 genome. The promoter works in the presence of inorganic phosphate and glucose. In this study, we present the essential region of the scmp promoter (promoter $\mathrm{C}$ ), which lacks $358 \mathrm{bp}$ of the 5 ' region of the full-length promoter. Promoter $\mathrm{C}$ was very short and contained only $63 \mathrm{bp}$. Moreover, there was no restriction on the medium component by combining the promoter and the S. livians 1326 strain. Using promoter $C$ and $S$. lividans, we succeeded in the extracellular production of the Streptomyces enzymes leucine aminopeptidase, ferulic acid esterase, and transglutaminase, which possessed signal peptides for secretion via the second pathway, at high levels.

\section{Introduction}

Actinomycetes are gram-positive bacteria that are useful tools for the biological production of several secondary metabolites, including antibiotics. Actinomycetes are also known to produce various enzymes, such as proteases (Morihara et al. 1969; Hatanaka et al. 2005; Uesugi et al. 2011), cellulase, and hemicellulase (Kumar et al. 2020). In recent years, genome analysis technology has been developed, and there are many opportunities to select and express these useful proteins from genomic information. However, the production of recombinant proteins from actinomycetes using Escherichia coli as a host sometimes results in the failure to form inclusion bodies. One of the reasons for this is GC-rich (ca. 70\%) in their genomes. Actinomycetes have a good ability to secrete proteins into the culture medium because they do not have an outer membrane. Thus, Actinomyces are a suitable host for the production of secreted proteins from actinomycetes (Anné et al. 1993; Anné et al. 2014). We have previously reported a metalloendoprotease (SCMP) from Streptomyces cinnamoneus (previously named S. septatus) TH-2 strain (2). SCMP was highly expressed and secreted using a medium that was rich in glucose and inorganic phosphate, and the yield was $0.3 \mathrm{~g} / \mathrm{L}$ (2). To utilize this hyperproduction, we cloned the upstream region of the scmp gene as a promoter and created an expression vector of pTONA5a, which was based on pIJ702, for expression in S. lividans. The vector harbors ori and rep for streptomycetes and thiostrepton-resistant genes (Hatanaka et al. 2008). By combining this vector with the S. lividans 1326 strain, we succeeded in the extracellular production of several Streptomyces enzymes (Hatanaka et al. 2008; Uraji et al. 2018; Wan et al. 2019). In this study, we attempted to identify an essential region of the scmp promoter to enhance its expression. We confirmed that the truncated promoter showed higher protein expression than the full-length promoter. Furthermore, we succeeded in protein expression by using the truncated promoter in medium that was not rich in glucose and phosphate and in commercial tryptic soy broth (TSB).

\section{Materials And Methods}




\section{Culture conditions and transformation of S. lividans}

Escherichia coli JM109 was used as the host cell for vector construction. S. cinnamoneus TH-2 was used as the source of the promoter. We used a secreted leucine aminopeptidase (LAP) from S. griseus as a reporter gene and the S. lividans 1326 strain as an expression host. The PG medium contained $2.0 \%$ glucose, $0.8 \% \mathrm{~K}_{2} \mathrm{HPO}_{4}, 0.05 \% \mathrm{MgSO}_{4} \cdot 7 \mathrm{H}_{2} \mathrm{O}, 0.5 \%$ polypeptone, and $0.5 \%$ yeast extract. In the $0.1 \%$ Pglu medium, the $\mathrm{K}_{2} \mathrm{HPO}_{4}$ concentration was changed to $0.1 \%$ in $\mathrm{PG}$ medium, and glucose was replaced with glycerol in PG medium in the Pgly medium. Tryptic soy broth was purchased from Japan Becton, Dickinson, and Company (Tokyo, Japan). Protoplast preparation and transformation were performed according to the method described by Hopwood et al. (Hopwood et al. 1985). The constructed plasmids were transformed into protoplasts of the S. lividans 1326 strain, and transformants were selected by thiostrepton resistance. The obtained transformants were inoculated in LB medium containing $1.0 \%$ trypton, $0.5 \%$ yeast extract, and $1.0 \% \mathrm{NaCl}$ and cultured until the stationary phase. The cells were then collected and washed with $0.9 \% \mathrm{NaCl}$, and the cells were inoculated into the medium.

\section{Construction of expression plasmids containing the truncated scmp promoters}

The pTONA5a plasmid with the leucine aminopeptidase gene (pTONA5a-lap) was constructed as described previously (Hatanaka et al. 2008). PCR was performed to obtain genes with truncated promoters. To amplify the fragments of $A, C$, and $D$, the forward primers with Asel site (ATTAAT) were constructed at the $-277 \mathrm{bp},-66 \mathrm{bp}$, and $-46 \mathrm{bp}$ sites of scmp, and the reverse primers were constructed at the end of the inserted genes with HindIII. The fragment of A was amplified with the following primers: $5^{\prime}-$ ATTAATCGGCAACCAGCCGCCGACGG-3' (the underlying indicates an Asel site) and 5'AAGCTTAGGTGGGCGGTTCGCCGGTG-3' (the underling indicates a Hindlll site). The fragment of $C$ was amplified with the following primers: 5'- ATTAATCACCACACCTGCACCACAGG-3' (the underling indicates an Asel site) and 5'-AAGCTTAGGTGGGCGGTTCGCCGGTG-3' (the underling indicates a HindIII site). The fragment of $D$ was amplified with the following primers: 5 '- ATTAATGCGGTCGTACAACCGGCTGC-3' (the underlying indicates an Asel site) and 5'-AAGCTTAGGTGGGCGGTTCGCCGGTG-3' (the underlying indicates a Hindlll site). The fragment of $B$ was amplified with the following primers: $5^{\prime}-$ ATTAATCGGCAACCAGCCGCCGACGG-3' (the underlying indicates an Asel site) and 5'CATATGTGTCTCCTATGAGGGGGGTTGG-3' (the underlying indicates an Ndel site). The pTONA5a-lap plasmid was used as the template. The amplified fragments were cloned in pCR-Blunt II-TOPO (Thermo Fisher Scientific, Tokyo, Japan), and the inserted sequences were confirmed. Clones A, C, and D were digested with Asel and HindIII, and the fragments were ligated into the Asel-HindIII gaps of pTONA5. Clone B was digested with Asel and Ndel, and the fragment was ligated into the Asel-Ndel gap of PTONA5.

\section{Construction of expression plasmids for ferulic acid esterase (FAE) and transglutaminase (TG)}

The FAE gene from S. cinnamoneus NBRC12852 was cloned and amplified with the following primers: 5'CATATGATCATGATCAGCTCGATGAGGAAGACATC-3' (the underling indicates an Ndel site) and 5'- 
AAGCTTCACTTCATGAAGGTGACCTCGGGGT-3' (the underlying indicates a Hindlll site). The PCR product was cloned into PCR-Blunt II-TOPO, and cloning was confirmed by sequencing. The resulting FAE gene was cloned into the gap of Ndel-Hindlll of pTONA containing promoter C. TG construction from $S$. mobaraensis NBRC13864 has already been reported (Tokai et al. 2020).

\section{Assay of LAP activity}

LAP activity was determined using Leu-pNA (Sigma-Aldrich Japan, Tokyo, Japan) as a substrate. The sample $(0.1 \mathrm{~mL})$ and $0.1 \mathrm{~mL}$ of the substrate $(32 \mathrm{mM})$ were added to $0.8 \mathrm{~mL}$ of a $100 \mathrm{mM}$ Tris- $\mathrm{HCl}(\mathrm{pH}$ 8.0) and $1 \mathrm{mM} \mathrm{CaCl}_{2}$ solution. The amount of $p$-nitroaniline released was determined by measuring the absorbance at $405 \mathrm{~nm}$ using a spectrophotometer (UV2800, Hitachi Ltd.). The initial velocity was determined from the linear region of the optical density profile $\left(\varepsilon 405 \mathrm{~nm}=10,600 \mathrm{M}^{-1} \mathrm{~cm}^{-1}\right)$ (Arima et al. 2006).

\section{DNA accession numbers.}

The accession numbers assigned to the sequences in the DNA Data Bank of Japan (DDBJ) database are as follows: SCMP, AB189036; LAP, AB125217; FAE, AB921569; and TG, AY615816.

\section{Results}

\section{LAP activity and expression by full-length scmp promoter in PG medium}

We have reported that SCMP, which is a metalloendoprotease from S. cinnamoneus $\mathrm{TH}-2$ strain controlled under the scmp promoter, required $2 \%$ glucose and $0.8 \%$ phosphate to be secreted into the culture as an active form (Hatanaka et al. 2005). Based on our findings, we examined four media for secreted LAP expression using the transformant harboring the pTONA5a-lap. After 4 days of culture at $30^{\circ} \mathrm{C}$, the LAP activity of the supernatant was analyzed, and protein expression was detected by SDS-PAGE. The supernatant of the culture in PG medium showed LAP activity and protein expression (Fig. 1). In contrast, low phosphate medium $\left(0.1 \% \mathrm{~K}_{2} \mathrm{HPO}_{4}\right.$ in $\mathrm{PG}$ ), Pgly medium ( $2 \%$ glycerol instead of glucose in $\mathrm{PG}$ ), and TSB showed lower LAP activity and expression than PG medium (Fig. 1).

\section{LAP activity and expression by truncated scmp promoters}

To identify the essential region of the scmp promoter, we constructed expression vectors combined with truncated promoters and the lap gene (Fig. 2). pTONA5a-lap, which included the full-length scmp promoter ( $F L)$, and expression vectors with four types of truncated scmp promoters (A, B, C, and D) were transformed into the $S$. lividans 1326 strain, and the obtained transformants were cultivated in PG medium. As shown in Fig. 3A, the A promoter, which lacks 148 bp of the 5 ' region of the scmp promoter, showed three-fold higher LAP activity than FL. In addition, promoter C, which lacked 358 bp of the $5^{\prime}$ region of the scmp promoter, showed four-fold higher activity than that of FL. Promoter $D$, which harbors 46 bp of the 3 ' region in the scmp promoter, also showed two-fold higher LAP activity than FL. Promoter B 
lacked $206 \mathrm{bp}$ of the 3 region of the scmp promoter. The secreted LAP proteins were detected except for promoter B, and the content of LAP proteins increased in order from A and C to D compared with FL (Fig. 3B).

\section{Truncated promoter $\mathrm{C}$ required no glucose and phosphate for lap expression}

From the results of the truncated promoter in PG medium (Fig. 3), we expected that promoter C would require no glucose and phosphate and would be easy to use. Thus, we compared the secreted LAP activities using $\mathrm{FL}$ and truncated promoters $\mathrm{A}$ and $\mathrm{C}$ under the conditions of low phosphate concentration ( $0.1 \%$ phosphate), the exchanged carbon source of glucose with glycerol (Pgly), and TSB. The LAP activity using the FL promoter was the highest in the PG medium compared to the other three cultivation conditions (Fig. 4A). The LAP activity of promoter A was remarkably increased in the low-phosphate medium. This value was three times higher than that of FL. Furthermore, the LAP activity of promoter $C$ was higher than that of FL and A under all conditions (Fig. 4A). Moreover, the protein expression levels under the four conditions by the $C$ promoter were also increased compared with those under the $\mathrm{FL}$ promoter under PG (Fig. 4B). In particular, the LAP protein was strongly secreted by the combination of the $C$ promoter and Pgly medium (Fig. 4B). In addition, the levels of other secreted proteins were reduced by using promoters $C$ and TSB, although the content of secreted LAP protein was slightly lower than that in PG or Pgly (Fig. 4B).

\section{Expression of other actinomyces proteins by promoter $\mathrm{C}$}

We attempted to produce other proteins with promoter C. FAE is an enzyme that releases ferulic acid from plant biomass (Uraji et al. 2013). When the Streptomyces FAE gene (Uraji et al. 2018) was cloned downstream of promoter $\mathrm{C}$ and expressed in $\mathrm{PG}$ and TSB media, enzyme production was confirmed in both (Fig. 5). To utilize the full-length scmp promoter, it was necessary to use PG medium; however, when cultured in PG medium, the expression of proteins other than the target protein was induced, and multiple proteins were often expressed simultaneously. It was necessary to purify the culture supernatant and extract only the target protein. In the case of FAE expressed by promoter $\mathrm{C}$, the expression of contaminating proteins was suppressed in both PG and TSB medium, and the expression of other proteins was particularly reduced in TSB medium (Fig. 5). TG mainly connects proteins (glutamine side chain) to proteins (lysine side chains) by covalent bonds; by utilizing this property, it can be used in the processing of marine products, modification of meat, and processing of dairy products (Tokai et al. 2020). Similar to the FAE, TG was expressed by promoter $C$ in $P G$ or other media. The enzyme is released into the culture's supernatant due to its secretory signal; there is a prosequence downstream of the secretory signal, which is cleaved by another enzyme (peptidase) secreted after TG. By removing the prosequence, the enzyme is converted into an active mature form of TG. When TG was expressed by promoter C in PG medium, a mature protein mixed with the prosequence form was expressed (Fig. 5). On the other hand, when TG was expressed in Pgly medium, conversion to the mature form of TG was completed. These results indicate that using promoter $C$ facilitates the secretion of several types of proteins because the promoter does not restrict the carbon source. 


\section{Discussion}

In this study, we identified the essential region (63 bp, promoter $\mathrm{C}$ ) of the scmp promoter. By using pTONA containing promoter $\mathrm{C}$ and $\mathrm{S}$. lividans, we succeeded in the extracellular expression of three Streptomyces enzymes, LAP, FAE, and TG, which possessed signal peptides for secretion via the second pathway.

The full-length scmp promoter consists of $424 \mathrm{bp}$ upstream sequences of the SCMP gene in the $S$. cinnamoneus $\mathrm{TH}-2$ genome. To date, we have not determined the sequence similarity to the genome information of other organisms or actinomycetes. Because the promoter is powerful, it is used to commercially produce two Streptomyces enzymes. However, there is a restriction on the medium component of enzyme expression using the promoter. This study aimed to remove the restriction on the use of the scmp promoter by identifying the essential region. We presented the essential region of the scmp promoter (promoter $\mathrm{C}$ ), which lacks $361 \mathrm{bp}$ of the 5 ' region of the full-length promoter. Promoter $\mathrm{C}$ was very short and consisted only of $63 \mathrm{bp}$. Moreover, there was no restriction of the medium component by the combination of the promoter and the $S$. livians 1326 strain. This system will be useful for producing and characterizing streptomycete proteins.

In Streptomyces, protein expression in secondary metabolism and morphological changes are controlled by several transcriptional factors (Romero-Rodriguez et al. 2015). The scmp gene is homologous to sgmA genes that encode a metalloendoprotease (SGMP) involved in morphological changes in Streptomyces griseus, whose expression is controlled by AdpA (Ohnishi et al. 2005). AdpA is an important transcriptional regulator that binds to DNA, including the motif -TGGCSNGWWY- (Ohnishi et al. 2005). Kato et al. reported the loss of AdpA binding ability in the sgmA promoter region led to the loss of SGMP protein expression (Kato et al. 2002). First, we estimated that the scmp promoter also controlled the AdpA protein or a similar regulation system of AdpA. We found four AdpA-binding motif-like sequences, CTGCCCGAAC-, -AGGTGCGTTT-, -ATGCCCGAAC-, and -CGTCCGGTAT-, in approximately $250 \mathrm{bp}$ of the scmp promoter. However, the truncated scmp promoters $C$ and $D$, which lacked more than 300 bp in the $5^{\prime}$ region of the full-length scmp promoter, were active (Fig. 3). These results suggest that the working mechanism of the scmp promoter is different to that of SGMP by AdpA.

The feature of the scmp promoter is that a large amount of protein is secreted extracellularly. By using PG medium, the content of secreted proteins reached the maximum at least 4 days of culture, this cultivation time resulting in the secretion of many proteins, which might be produced by secondary metabolism (Fig. 3B). Using promoters $\mathrm{C}$ and $\mathrm{TSB}$, the content of secreted proteins was more intense in the target protein of LAP than in PG medium (Fig. 4B). It was indicated that promoter $\mathrm{C}$ might be regulated differently to be compared with the full-length promoter. Further research is needed to reveal how the promoter functions in streptomycetes.

Because the medium component was not restricted, extracellular production of recombinant proteins using the truncated promoter $\mathrm{C}$ was more convenient than the full-length promoter. In Fig. 5, we represented FAE and TG expression using promoter $\mathrm{C}$ in $\mathrm{PG}$ and other media. By using promoter $\mathrm{C}$ and the PG medium, this conversion was insufficient, and zymogen and active TG coexisted in the culture 
(Fig. 5(B)). The conversion was complete using promoter $\mathrm{C}$ and Pgly media. From these results, it can be inferred that the expression of the host peptidase was active in the Pgly medium, and conversion to the mature form was performed more rapidly than that in the TSB and PG media. This result suggests that it was difficult to select various media with the conventional full-length scmp promoter, but by using the 63base promoter, the medium's components were no longer restricted and the enzyme to be expressed was expanded.

Promoter $\mathrm{C}$ is very short and convenient, exerting no restriction on the medium component. A similar short promoter of kasOp* (97 bp) from S. coelicolor has been reported. The promoter was engineered by random mutagenesis between sites -10 and -35 , and screened using the Lux reporter. The kasOp* exhibited showed higher activity at the transcription and protein levels than those of known strong promoters of ermEp* and SF14p (Wang et al. 2013). Further work is in progress to compare the activities of promoter $\mathrm{C}$ and the kasOp ${ }^{*}$ promoter in Streptomyces protein expression.

\section{Conclusions}

In this study, we present the essential region of the scmp promoter (promoter C), which lacks $361 \mathrm{bp}$ of the $5^{\prime}$ region of the full-length promoter. Promoter $\mathrm{C}$ was very short and only consisted of $63 \mathrm{bp}$. Moreover, there was no restriction on the medium component by the combination of the promoter and the $S$. livians 1326 strain. This system will be a useful tool for producing and characterizing streptomycetes proteins.

\section{Abbreviations}

\section{SCMP}

metalloendoprotease from Streptomyces cinnamoneus

TSB

tryptic soy broth

LAP

leucine aminopeptidase

FAE

ferulic acid esterase

TG

transglutaminase.

\section{Declarations}

\section{Acknowledgement}

We thank Mr. Ryota Nakahigasi (Nagase ChemteX Corporation) for providing the protoplasts of $S$. lividans.

\section{Authors' contributions}


$\mathrm{MU}$ and TH constructed the plasmids. MU and $\mathrm{LY}$ conducted expression experiments. MU and TH wrote the manuscript. All authors read and approved the final manuscript.

\section{Funding}

This work was supported by the Japan Society for the Promotion of Science (grant number 26420805 to $\mathrm{TH})$.

\section{Availability of data and materials}

All data generated or analyzed during this study are included in this published article.

\section{Ethics approval and consent to participate}

Not applicable.

\section{Consent for publication}

Not applicable.

\section{Competing interests}

The authors declare that they have no competing interests.

\section{Author details}

${ }^{1}$ Research Institute for Biological Sciences (RIBS), Okayama, 7549-1 Kibichuo-cho, Kaga-gun, Okayama, 716-1241, Japan.

2 Present address: Graduate School of Science, Technology and Innovation, Kobe University, 1-1, Rokkodai-cho, Nada-ku, Kobe 657-8501, Japan.

\section{References}

1. Anné J, Van Mellaert L (1993) Streptomyces lividans as host for heterologous protein production. FEMS Microbiol Lett 114(2):121-128

2. Anné J, Vrancken K, Van Mellaert L, Van Impe J, Bernaerts K (2014) Protein secretion biotechnology in Gram-positive bacteria with special emphasis on Streptomyces lividans. Biochim Biophys Acta 1843(8):1750-1761

3. Arima J, Uesugi Y, Iwabuchi M, Hatanaka T (2006) Study on peptide hydrolysis by aminopeptidases from Streptomyces griseus, Streptomyces septatus and Aeromonas proteolytica. Appl Microbiol Biotechnol 70:541-547

4. Hatanaka T, Uesugi Y, Arima J, Iwabuchi M (2005) Purification, characterization cloning, and sequencing of metalloendopeptidase from Streptomyces septatus TH-2. Arch Biochem Biophys 
434:289-298

5. Hatanaka T, Onaka H, Arima J, Uraji M, Uesugi Y, Usuki H, Nishimoto Y, Iwabuchi M (2008) pTONA5: a hyperexpression vector in streptomycetes. Protein Expr Purf 62:244-248

6. Hopwood DA, Bibb MJ, Chater KF, Kieser T, Bruton CJ, Kieser HMLydiate DJ

7. Smith CP, Ward H, Schrempf CJ (1985) A laboratory manual. The John Ines Foundation, Norwich, pp $70-84$

8. Kato J, Suzuki A, Yamazaki H, Ohnishi Y, Horinouchi S (2002) Control by A- factor of a metalloendopeptidase gene involved in aerial mycelium formation in Streptomyces griseus. $\mathrm{J}$ Bacteriol 184(21):6016-6025

9. Kumar M, Kumar P, Das P, Solanki R, Kapur M (2020) Potential applications of extracellular enzymes from Streptomyces spp. in various industries. Arch Microbiol 202(7):1597-1615

10. Morihara K, Tsuzuki T, Oka T (1969) Comparison of the specificities of various serine proteinases from microorganisms. Arch Biochem Biophys 129: 620-634 (1969)

11. Ohnishi Y, Yamazaki H, Kato J, Tomono A, Horinouchi S (2005) AdpA, a central transcriptional regulator in the A-factor regulatory cascade that leads to morphological development and secondary metabolism in Streptomyces griseus. Biosci Biotechnol Biochem 69(3):431-439

12. Romero-Rodríguez A, Robledo-Casados I, Sánchez S (2015) An overview on transcriptional regulators in Streptomyces. Biochim Biophys Acta 1849(8):1017-1239

13. Tokai S, Uraji M, Hatanaka T (2020) Molecular insights into the mechanism of substrate recognition of Streptomyces transglutaminases. Biosci Biotechnol Biochem 84(3):575-582

14. Uesugi $Y$, Usuki $H$, Iwabuchi M, Hatanaka T (2008) Highly potent fibrinolytic serine protease from Streptomyces. Enzyme Microb Technol 48(1):7-12

15. Uraji M, Kimura M, Inoue Y, Kawakami K, Kumagai Y, Harazono K, Hatanaka T (2013) Enzymatic production of ferulic acid from defatted rice bran by combination of bacterial enzymes. Appl Biochem Biotechnol 171(5):1085-1093

16. Uraji M, Tamura H, Mizohata E, Arima J, Wan K, Ogawa K, Inoue T, Hatanaka T (2018) Loop of Streptomyces feruloyl esterase plays an important role in the enzyme's catalyzing the release of ferulic acid from biomass. Appl Environ Microbiol 84(3): e02300-17 (2018)

17. Wan K, Uraji M, Tokai S, Hatanaka T (2019) Enzymatic degradation of allergen peptides from bovine casein by a combination of Streptomyces Aminopeptidases. Appl Biochem Biotechnol 187(2):570582

18. Wang W, Li X, Xiang A, Feng X, Yang K (2013) An engineered strong promoter for streptomycetes. Appl Environ Microbiol 79(14): 4484-4492 (2013)

\section{Figures}


(A)

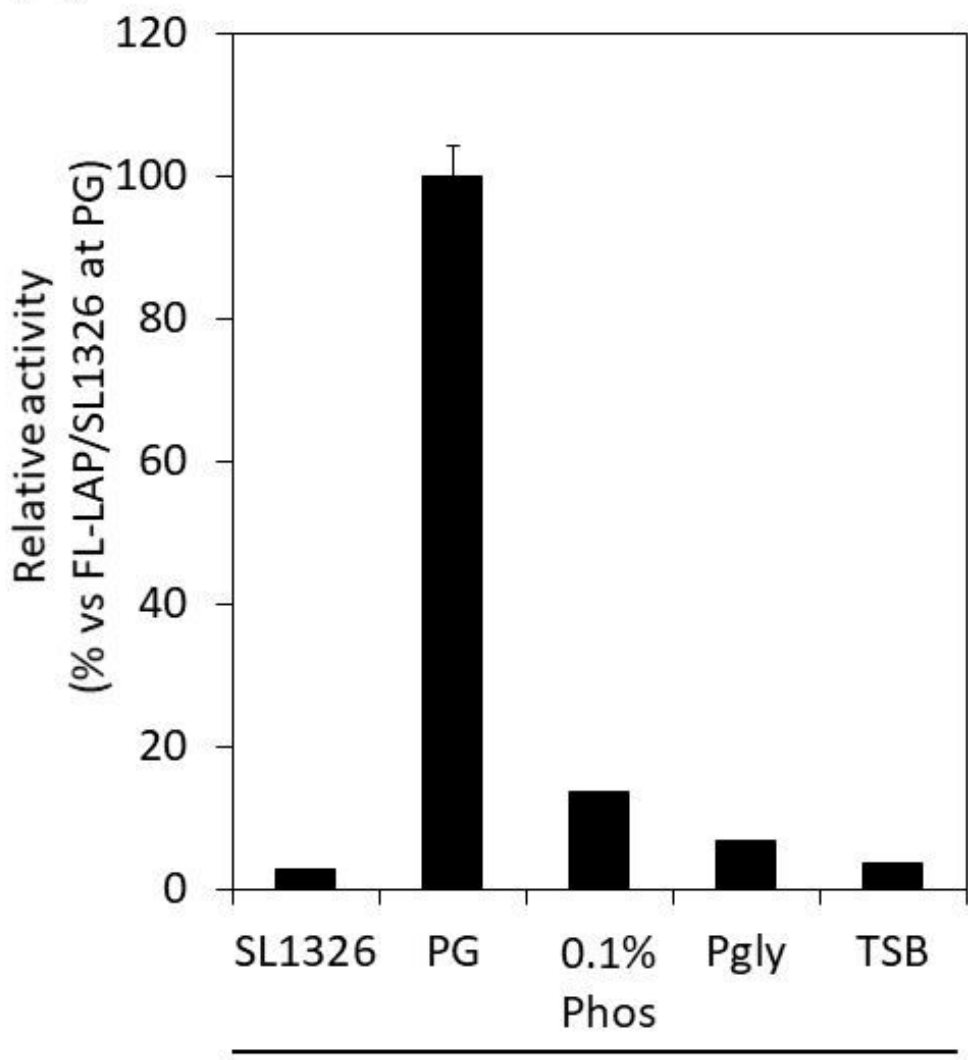

FL-LAP/SL1326
(B)

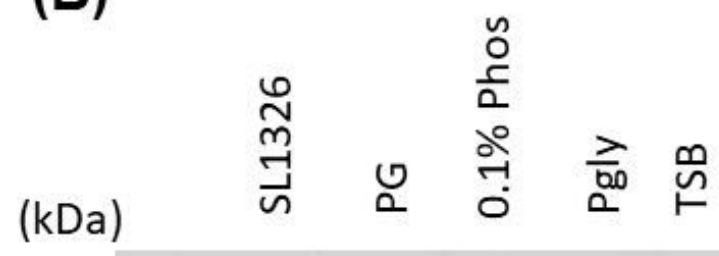

80

40

30

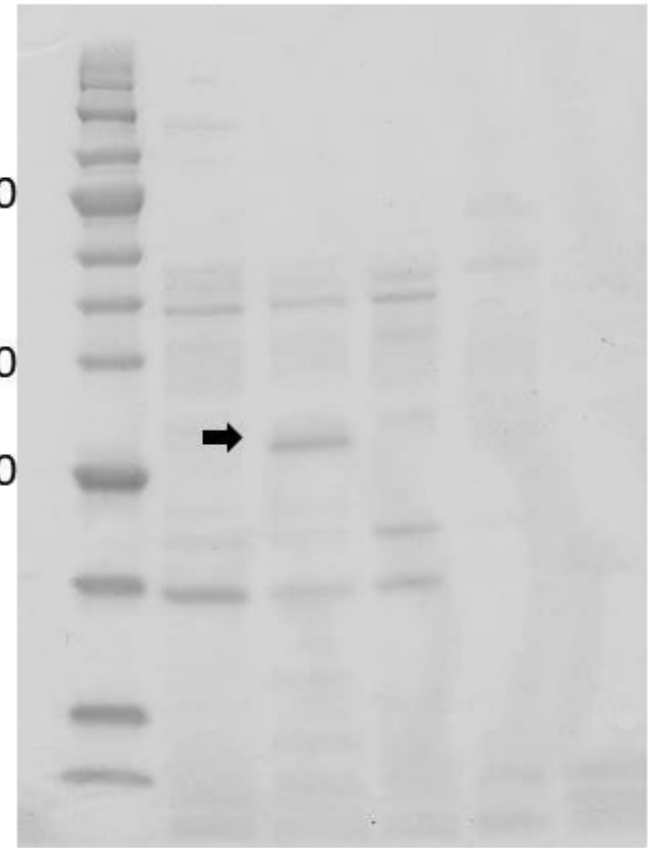

Fig. 1

\section{Figure 1}

LAP activities and expression by the full-length scmp promoter.

(A) LAP activities by the promoter cultured in different media. (B) SDS-PAGE analysis of LAP expression by the promoter cultured in different media. The gel used was $10 \%$ polyacrylamide and was stained with Coomassie Brilliant Blue. All lanes were loaded with $10 \mu \mathrm{L}$ of the culture supernatant. Arrows indicate the locations of the LAP proteins. 


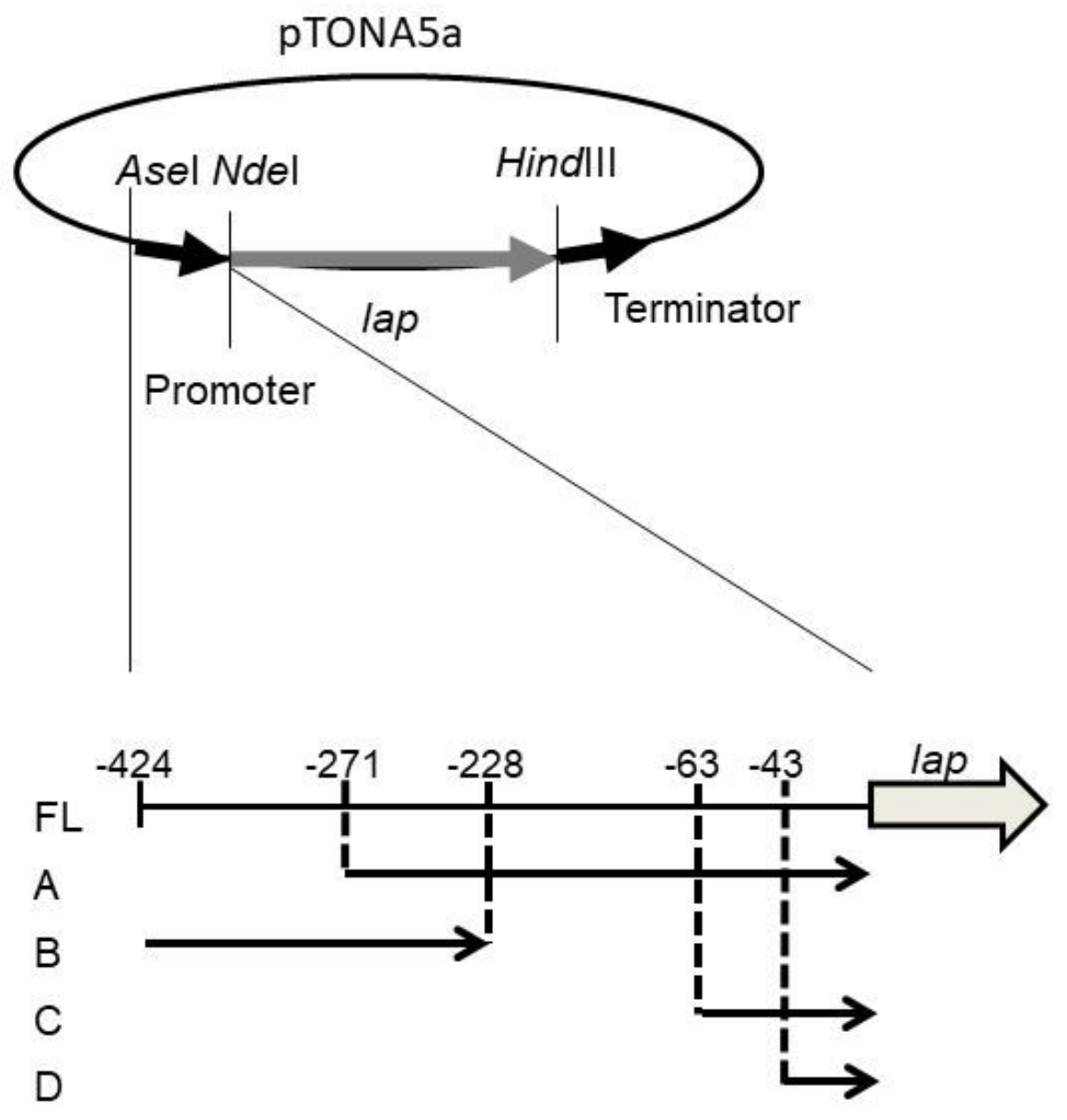

Fig. 2

Figure 2

Schematic representation of pTONA5a plasmid construction, including truncated SCMP promoters and the lap gene; promoter; scmp promoter, terminator; kibilysin terminator, lap; leucin aminopeptidase, FL; full length scmp promoter. 
(A)

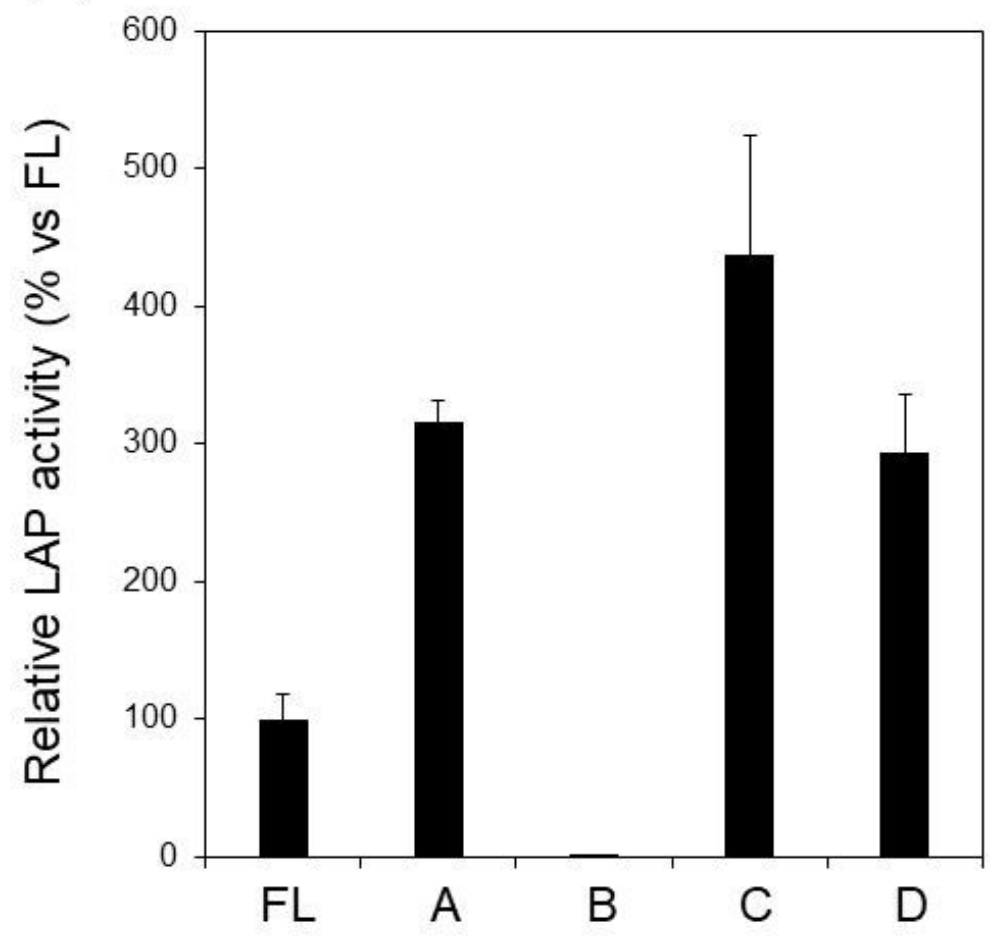

(B)

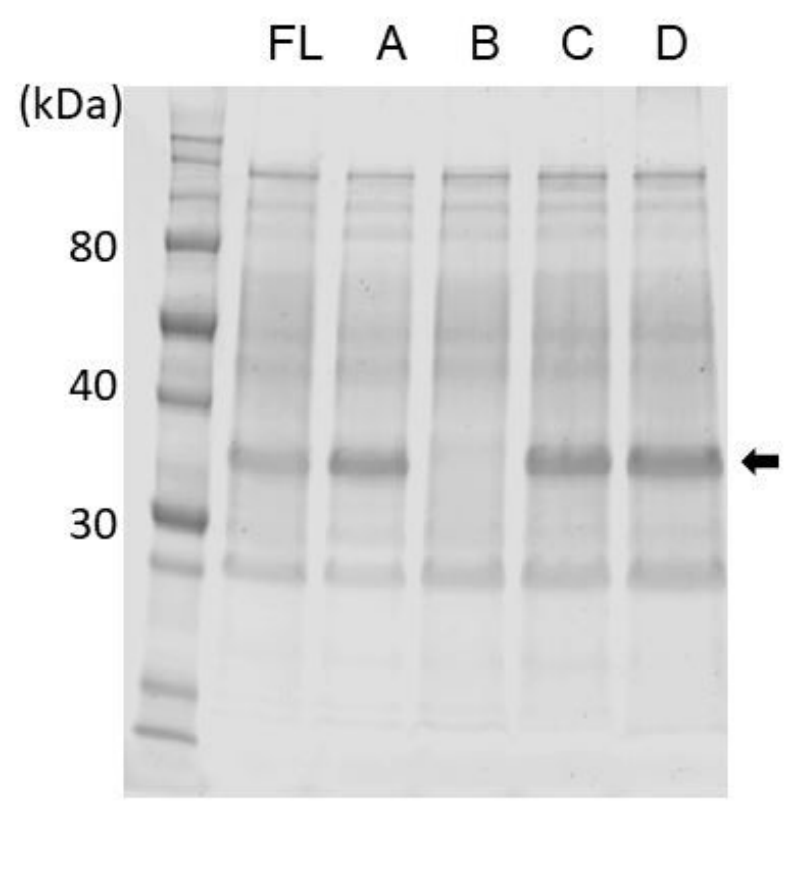

Fig. 3

\section{Figure 3}

LAP activities and expression using SCMP promoters and PG medium.

(A) Comparison of LAP activities in culture supernatants of the full length (FL) and truncated (A-D) scmp promoters. The activity was normalized to the FL values. The average value from three independent experiments is shown. Error bars represent the SE. (B) SDS- PAGE analysis of culture supernatants by FL and A-D truncated scmp promoters. The gel used was $10 \%$ polyacrylamide and was stained by Coomassie Brilliant Blue. All lanes were loaded with $10 \mu \mathrm{L}$ of culture supernatant. Arrows indicate the locations of the LAP proteins. 
(A)

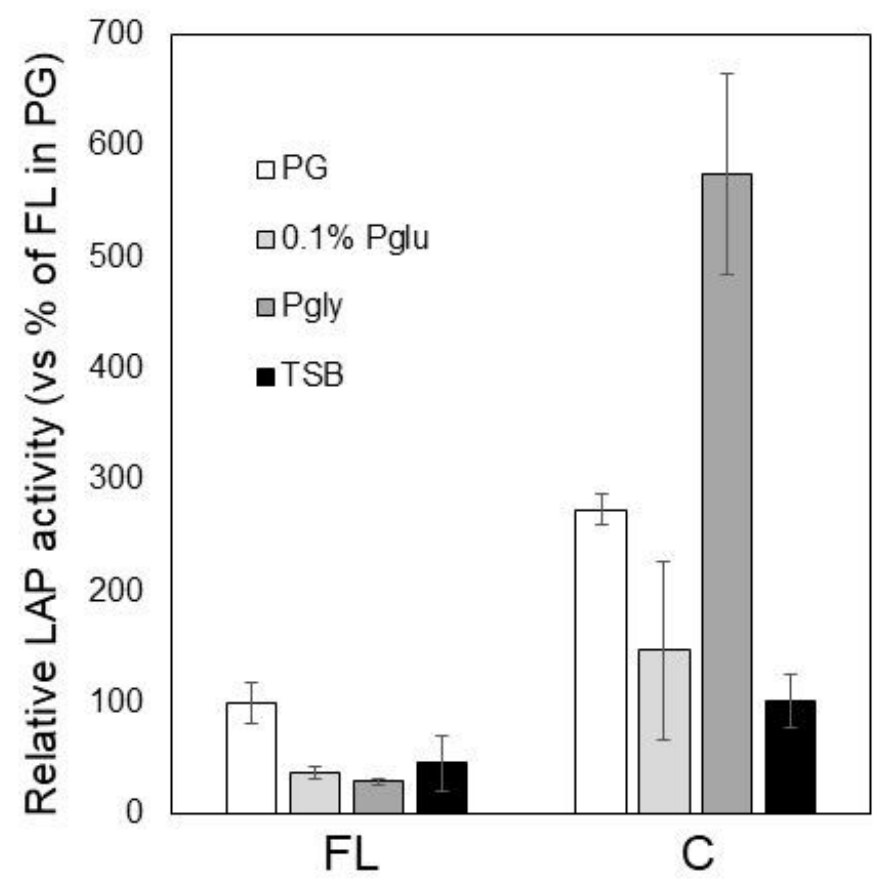

(B)

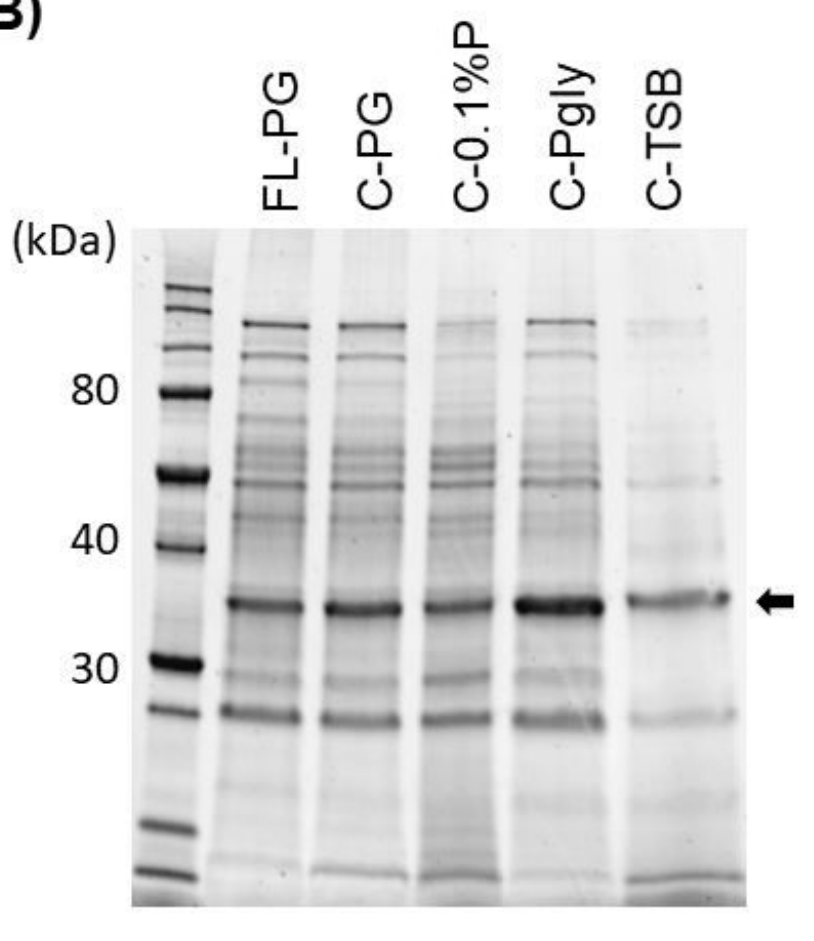

Fig. 4

Figure 4

LAP activities and expression of SCMP promoters in different media.

(A) Comparison of LAP activities in culture supernatants of full length $(F L)$ and truncated promoter $C$ in different media. PG; PG medium (0.8\% $\mathrm{KH}_{2} \mathrm{PO}_{4}$ and $2 \%$ glucose), $0.1 \%$ Pglu; $0.1 \% \mathrm{KH}_{2} \mathrm{PO}_{4}$ and $2 \%$ glucose, Pgly; $0.8 \% \mathrm{KH}_{2} \mathrm{PO}_{4}$, and $2 \%$ glycerol. The activity was normalized to that of $\mathrm{FL}$ in PG medium values. The average value from three independent experiments is shown. Error bars represent the SE. (B) SDS-PAGE analysis of culture supernatants with FL in PG medium and $\mathrm{C}$ truncated scmp promoters in different media. The gel used was $10 \%$ polyacrylamide and was stained by Coomassie Brilliant Blue. All lanes were loaded with $10 \mu \mathrm{L}$ of culture supernatant. 
(A)

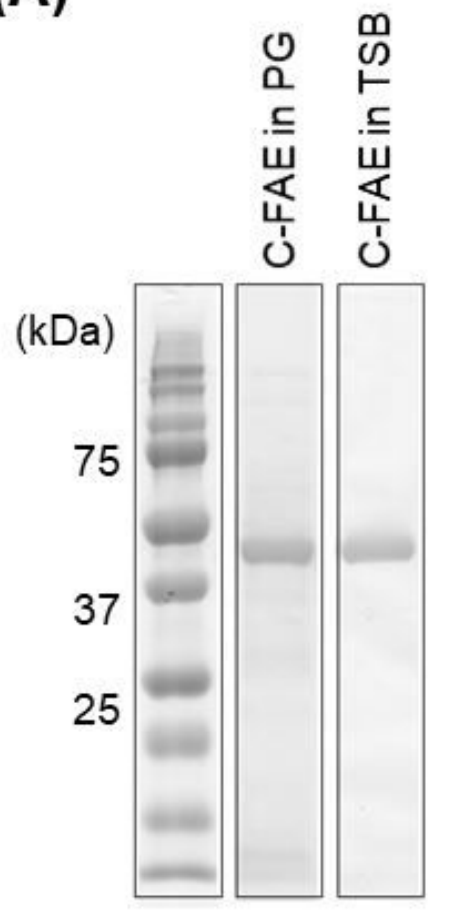

(B)

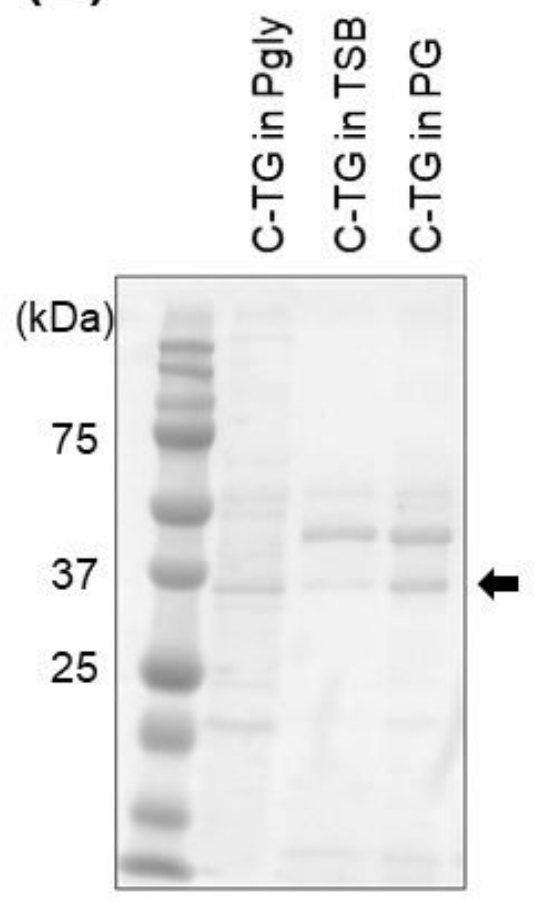

Fig. 5

\section{Figure 5}

FAE and TG expression by promoter $\mathrm{C}$ in different media

(A) FAE expression by promoter $\mathrm{C}$ in PG and TSB media. (B) TG expression by promoter C in PG, TSB, and Pgly media. The gel used was $10 \%$ polyacrylamide and was stained by Coomassie Brilliant Blue. All lanes were loaded with $10 \mu \mathrm{L}$ of culture supernatant. An arrow indicates the mature form of TG protein.

\section{Supplementary Files}

This is a list of supplementary files associated with this preprint. Click to download.

- Graphicabstract.tif 